Research Article

\title{
Characterization of Nonstationary Phase Noise Using the Wigner-Ville Distribution
}

\author{
Chagai Levy $(\mathbb{D}$, Monika Pinchas $(\mathbb{D}$, and Yosef Pinhasi $(\mathbb{D}$ \\ Department of Electrical and Electronic Engineering, Ariel University, Ariel 40700, Israel \\ Correspondence should be addressed to Chagai Levy; chagailevy@gmail.com
}

Received 6 September 2019; Revised 26 February 2020; Accepted 12 March 2020; Published 20 April 2020

Guest Editor: Filipe J. Marques

Copyright (c) 2020 Chagai Levy et al. This is an open access article distributed under the Creative Commons Attribution License, which permits unrestricted use, distribution, and reproduction in any medium, provided the original work is properly cited.

\begin{abstract}
Oscillators and atomic clocks, as well as lasers and masers, are affected by physical mechanisms causing amplitude fluctuations, phase noise, and frequency instabilities. The physical properties of the elements composing the oscillator as well as external environmental conditions play a role in the coherence of the oscillatory signal produced by the device. Such instabilities demonstrate frequency drifts, modulation, and spectrum broadening and are observed to be nonstationary processes in nature. Most of the tools which are being used to measure and characterize oscillator stability are based on signal processing techniques, assuming time invariance within a temporal window, during which the signal is assumed to be stationary. This letter proposes a new time-frequency approach for the characterization of frequency sources. Our technique is based on the Wigner-Ville timefrequency distribution, which extends the spectral measures to include the temporal nonstationary behavior of the processes affecting the coherence of the oscillator and the accuracy of the clock. We demonstrate the use of the technique in the characterization of nonstationary phase noise in oscillators.
\end{abstract}

\section{Introduction}

In recent years, due to the high data rates required to be transferred in communication networks, the demand for highaccuracy satellite navigation systems and the development of high-resolution radars, oscillators, and accurate frequency clocks with very high precision are required. Stable oscillators, including atomic ones such as rubidium and even cesium are often used in communication and navigation facilities [1,2]. Apart from the above applications, many other electronic systems require frequency generators. In such systems, time synchronization and an accurate frequency are required $[3,4]$.

Oscillators and clocks, as well as lasers and masers, are subjected to environmental conditions, internal malfunctions, and inherent physical phenomena causing instabilities in the oscillatory signal being produced [5]. These instabilities are expressed by amplitude and phase fluctuations causing frequency deviations, drift, and spectrum broadening. Instead of being stationary coherent, the generated signal is observed to be a stochastic process characterized by statistical and spectral features, which are nonstationary time-varying [6,7]. Therefore, their coherent features in the time and frequency domain should be investigated.

Phase noise is the frequency domain representation of rapid, short-term, and random fluctuations in the phase of a waveform. Historically, there have been two conflicting yet widely used definitions for phase noise. Some authors define phase noise to be the spectral density of a signal's phase only [8], while the other definition refers to the phase spectrum (which pairs up with the amplitude spectrum) resulting from the spectral estimation of the signal itself. The IEEE defines phase noise as $L(f)=S_{\varphi}(f) / 2$, where the "phase instability" is given in terms of the power spectral density $S_{\varphi}(f)$ of a signal's phase deviation $\varphi(t)$ defined for $f>0$ [9]. However, the standard phase noise [9] does not give the possibility of identifying and interpreting nonstationary effects.

In lasers and masers, the radiation line-width limit is calculated via the Schawlow-Townes expression [10-12], which assumes a stationary process [13-17] although it is not stationary in general.

In this letter, we propose a time-frequency metric for the characterization of oscillators and frequency generators, also 
considering nonstationary variations. It is based on the Wigner-Ville distribution $[18,19]$, proposed in 1932 for the characterization of quantum fluctuations. This time-frequency distribution is applicable for the analysis of nonstationary signals [20-23], radar signals [24, 25], biomedical signals [26-28], analysis of time-varying filters [29, 30], and image processing [31-33]. Up to now, the Wigner-Ville distribution was not yet used in the characterization of nonstationary oscillators such as evaluation of the phase noise. In this letter, we introduce an analytic expression of the Wigner-Ville timefrequency distribution for the characterization of the temporal coherency of nonstationary signals produced by classical and quantum oscillators. Our proposed expression can reveal the time-varying frequencies generally seen in the clock error noise under nonstationary conditions and in other platforms such as oscillator under vibrations or shocks.

The analysis is in general considering noise generated by different mechanisms. It is valid also for multinoise sources applied simultaneously to multinodes of the oscillator system. The multinoise sources are combination of two basic classes of noise, additive and parametric, in which their behavior and the underlying physical mechanisms are strikingly different. Each noise caused by an internal behavior of an oscillator or by a near-dc process modulates the carrier in phase.

\section{The Oscillator Model}

The model for the oscillator includes variation in the phase and given by $[5,34]$

$$
r(t) \simeq A \cos \left[2 \pi v_{0} t+\varphi(t)\right],
$$

where $A$ is the amplitude, $v_{0}$ is the central frequency of the oscillation, and $\varphi(t)$ represents the random fluctuation in phase. The phase variations $\varphi(t)$ is given as a summation of seven terms $[35,36]$ :

$$
\begin{aligned}
\varphi(t)= & 2 \pi v_{0}\left[x_{0}+y_{0} t+a \frac{t^{2}}{2}+\mu_{3} \frac{t^{3}}{6}+\sigma_{1} B_{H_{1}}(t)\right. \\
& \left.+\sigma_{2} \int_{0}^{t} B_{H_{2}}(s) d s+\sigma_{3} \int_{0}^{t} \frac{(t-s)^{2}}{2} d B_{H_{3}}(s)\right],
\end{aligned}
$$

where

(1) $x_{0}$ and $y_{0}$ are the initial phase and frequency offsets, respectively.

(2) $a$ is defined as the frequency drift coefficient.

(3) $\mu_{3}$ is a linear variation coefficient added to the frequency drift.

(4) $\left\{B_{H_{i}}(t), t \geq 0\right\}, i=1,2,3$ are three independent, onedimensional standard Wiener processes (Brownian motion) [35]. Following [37], the flicker noise is usually omitted from the mathematical model since it is not a rational process. The Wiener process is often referred to as an integral of white noise and is a nonstationary process.

(5) The constants $\sigma_{1}, \sigma_{2}$, and $\sigma_{3}$ represent the magnitude of the white frequency noise, the random walk frequency noise, and the frequency drift, respectively, which are three contributors to the noise in a typical oscillator. The constants $\sigma_{1}, \sigma_{2}$, and $\sigma_{3}$ are determined by the intensity of each of these respective noise components, resulting from internal electronic noise mechanisms in an oscillator, such as thermal (Johnson) noise, shot noise, and parametric noise, in which a near-dc process modulates the phase of the carrier [38].

(6) The frequency drift is a constant $\sigma_{3}=0$ [35].

The stochastic term of the phase variations $\varphi(t)$ is given by the term

$$
\Delta \varphi(t) \approx 2 \pi v_{0}\left[\sigma_{1} B_{H_{1}}(t)+\sigma_{2} \int_{0}^{t} B_{H_{2}}(s) d s\right],
$$

while the deterministic term of the phase is expressed as shift and drift in the oscillator frequency. Hereafter, we concentrate in the stochastic term (3) only and analyse its time-frequency characteristics using the Wigner-Ville distribution.

The stochastic term (3) is the phase noise causing broadening in the line width.

\section{The Wigner-Ville Distribution}

In this section, we will present briefly the Wigner-Ville distribution and its spectrum.

One of the most representative joint representations of time-frequency analysis is the Wigner-Ville distribution (WD), which is a quadratic signal representation introduced by Wigner in 1932 [19] and later applied by Ville in 1948 [39] for signal time-frequency analysis.

In the following, we will denote the expectation value of $x$ as $E\{x\}$.

The statistical autocorrelation of $\Delta \varphi(t)$ is denoted by $\bar{R}_{\Delta \varphi}(t, \tau)$ and defined as follows:

$$
\bar{R}_{\Delta \varphi}(t, \tau)=E\left\{\Delta \varphi(t+\tau / 2) \Delta \varphi^{*}(t-\tau / 2)\right\},
$$

where the shifting parameter is denoted by $\tau$.

The Wigner spectrum is defined as the Fourier transform of the statistical autocorrelation $\bar{R}_{\Delta \varphi}(t, \tau)$ [40]:

$$
\bar{W}_{\Delta \varphi}(t, f)=\int_{-\infty}^{\infty} \bar{R}_{\Delta \varphi}(t, \tau) e^{-i 2 \pi f \tau} d \tau
$$

where $f$ denotes the frequency variable.

\section{Time-Frequency Analysis of Phase Noise Using Wigner Spectrum}

This section proposes a new time-frequency closed form expression for the characterization of nonstationary phase noise in oscillators. Our technique is based on the Wigner spectrum, which extends the spectral measures to consist of the temporal nonstationary behavior of the phase process affecting the accuracy of the clock.

Theorem 1. We derive the Wigner spectrum (5) of the phase variation given in (3) and find that the closed form expression for a nonstationary phase noise is 


$$
\begin{aligned}
\bar{W}_{\Delta \varphi}(t, f)= & \left(2 \pi v_{0}\right)^{2}\left\{\frac{\sigma_{1}^{2}}{4(\pi f)^{2}}[1-\cos (4 \pi t f)]+\frac{\sigma_{2}^{2}}{(2 \pi f)^{4}}\right. \\
& -\frac{\sigma_{2}^{2}}{(2 \pi)^{4} f^{3}}\left[\frac{3}{f} \cos (4 \pi t f)+8 \pi t \sin (4 \pi t f)\right] \\
& \left.-\frac{\sigma_{2}^{2}}{2^{4} 3 f^{2}}\left[\cos (4 \pi t f)\left(\frac{3}{4 f^{2} \pi^{4}}-\frac{8 t^{2}}{\pi^{2}}\right)+\sin (4 \pi t f) \frac{4 t}{f \pi^{3}}\right]\right\} .
\end{aligned}
$$

Proof 1. By substituting $\Delta \varphi(t)$ (3) into $\bar{R}_{\Delta \varphi}(t, \tau)$ (4), we obtain

$$
\begin{aligned}
\bar{R}_{\Delta \varphi}(t, \tau)= & \left(2 \pi v_{0}\right)^{2} E\left\{\sigma_{1} B_{H_{1}}(t+\tau / 2)+\sigma_{2} \int_{0}^{t+\tau / 2} B_{H_{2}}(s) d s\right] \\
& \cdot\left[\sigma_{1} B_{H_{1}}^{*}(t-\tau / 2)+\sigma_{2} \int_{0}^{t-\tau / 2} B_{H_{2}}^{*}(s) d s\right\} .
\end{aligned}
$$

Because $\left\{B_{H_{i}}(t), t \geq 0\right\}, i=1,2$ are two independent, one-dimensional standard Wiener processes, we can write (7) as follows:

$$
\begin{aligned}
\bar{R}_{\Delta \varphi}(t, \tau)= & \left(2 \pi v_{0}\right)^{2} E\left\{\sigma_{1} B_{H_{1}}(t+\tau / 2) \sigma_{1} B_{H_{1}}^{*}(t-\tau / 2)\right. \\
& \left.+\sigma_{2} \int_{0}^{t+\tau / 2} B_{H_{2}}(s) d s \sigma_{2} \int_{0}^{t-\tau / 2} B_{H_{2}}^{*}(s) d s\right\} .
\end{aligned}
$$

$\varphi(t)$ is a real signal; therefore, $B_{H_{1}}^{*}(t)=B_{H_{1}}(t)$ and $B_{H_{2}}^{*}$ $(t)=B_{H_{2}}(t)$, and thus we can write $(8)$ as follows:

$$
\begin{aligned}
\bar{R}_{\Delta \varphi}(t, \tau)= & \left(2 \pi v_{0}\right)^{2} E\left\{\sigma_{1} B_{H_{1}}(t+\tau / 2) \sigma_{1} B_{H_{1}}(t-\tau / 2)\right. \\
& \left.+\sigma_{2} \int_{0}^{t+\tau / 2} B_{H_{2}}(s) d s \sigma_{2} \int_{0}^{t-\tau / 2} B_{H_{2}}(s) d s\right\} .
\end{aligned}
$$

The standard Wiener process $\left\{B_{H}(t), t \geq 0\right\}$ in its general form is a continuous-time Gaussian process on $[0, T]$, which starts at zero, has expectation zero for $t \in[0, T]$, and has the following covariance function [41, 42]:

$$
E\left\{B_{H}(t) B_{H}(s)\right\}=\frac{1}{2}\left(|t|^{2 H}+|s|^{2 H}-|t-s|^{2 H}\right) .
$$

In our case, the process $\left\{B_{H}(t), t \geq 0\right\}$ is the standard Brownian motion, i.e., $H=0.5$. By substituting (10) into (9), we obtain

$$
\begin{aligned}
\bar{R}_{\Delta \varphi}(t, \tau)= & \left(2 \pi v_{0}\right)^{2}\left\{\frac{\sigma_{1}^{2}}{2}(|t+\tau / 2|+|t-\tau / 2|-|\tau|)\right. \\
& \left.+E\left\{\sigma_{2} \int_{0}^{t+\tau / 2} B_{H_{2}}(s) d s \sigma_{2} \int_{0}^{t-\tau / 2} B_{H_{2}}(s) d s\right\}\right\} .
\end{aligned}
$$

Now, we will insert the expectation value into the joint integral argument on the second term of (11):

$$
\begin{aligned}
\bar{R}_{\Delta \varphi}(t, \tau)=\left(2 \pi v_{0}\right)^{2}\left\{\frac{\sigma_{1}^{2}}{2}(|t+\tau / 2|+|t-\tau / 2|-|\tau|)\right. \\
\left.\left.+\sigma_{2}^{2} \int_{0}^{t+\tau / 2} \int_{0}^{t-\tau / 2} E\left\{B_{H_{2}}(s) B_{H_{2}}\left(s^{\prime}\right)\right)\right\} d s d s^{\prime}\right\} .
\end{aligned}
$$

Next, we will preform the expectation value of the second term of (12) by using (10):

$$
\begin{aligned}
\bar{R}_{\Delta \varphi}(t, \tau)= & \left(2 \pi v_{0}\right)^{2}\left\{\frac{\sigma_{1}^{2}}{2}(|t+\tau / 2|+|t-\tau / 2|-|\tau|)\right. \\
& \left.+\sigma_{2}^{2} \int_{0}^{t+\tau / 2} \int_{0}^{t-\tau / 2} \frac{1}{2}\left(|s|+\left|s^{\prime}\right|-\left|s-s^{\prime}\right|\right) d s d s^{\prime}\right\} .
\end{aligned}
$$

Finally, we may write (13) in its final form:

$$
\begin{aligned}
\bar{R}_{\Delta \varphi}(t, \tau)= & \left(2 \pi v_{0}\right)^{2}\left\{\frac{\sigma_{1}^{2}}{2}(|t+\tau / 2|+|t-\tau / 2|-|\tau|)\right. \\
& +\frac{\sigma_{2}^{2}}{4}|t+\tau / 2|\left(t^{2}-\tau^{2} / 4\right)+\frac{\sigma_{2}^{2}}{4}|t-\tau / 2|\left(t^{2}-\tau^{2} / 4\right) \\
& \left.+\frac{\sigma_{2}^{2}}{12}\left[|\tau| \tau^{2}-|t+\tau / 2|(t+\tau / 2)^{2}-|t-\tau / 2|(t-\tau / 2)^{2}\right]\right\} .
\end{aligned}
$$

Then, we will turn to preform the closed form expression of $\bar{W}_{\Delta \varphi}(t, f)$ by substituting (14) into (5):

$$
\begin{aligned}
\bar{R}_{\Delta \varphi}(t, \tau)= & \left(2 \pi v_{0}\right)^{2}[a(t, \tau)+b(t, \tau)+c(t, \tau)+d(t, \tau) \\
& +e(t, \tau)+f(t, \tau)],
\end{aligned}
$$

where

$$
\begin{aligned}
& a(t, \tau)=\frac{\sigma_{1}^{2}}{2}(|t+\tau / 2|+|t-\tau / 2|-|\tau|), \\
& b(t, \tau)=\frac{\sigma_{2}^{2}}{4}|t+\tau / 2|\left(t^{2}-\tau^{2} / 4\right), \\
& c(t, \tau)=\frac{\sigma_{2}^{2}}{4}|t-\tau / 2|\left(t^{2}-\tau^{2} / 4\right), \\
& d(t, \tau)=\frac{\sigma_{2}^{2}}{12}|\tau| \tau^{2} \\
& e(t, \tau)=-\frac{\sigma_{2}^{2}}{12}|t+\tau / 2|(t+\tau / 2)^{2}, \\
& f(t, \tau)=-\frac{\sigma_{2}^{2}}{12}|t-\tau / 2|(t-\tau / 2)^{2} .
\end{aligned}
$$


Next, we will present the closed form expression for $\bar{W}_{\Delta \varphi}(t, f)$ by solving the Fourier transform of $(16)$ :

$$
\begin{aligned}
\bar{W}_{\Delta \varphi}(t, f) & =\left(2 \pi v_{0}\right)^{2} \int_{-\infty}^{\infty}[a(t, \tau)+b(t, \tau)+c(t, \tau)+d(t, \tau)+e(t, \tau)+f(t, \tau)] e^{-i 2 \pi f \tau} d \tau \\
& =\left(2 \pi v_{0}\right)^{2}[A(t, f)+B(t, f)+C(t, f)+D(t, f)+E(t, f)+F(t, f)],
\end{aligned}
$$

where $[A, B, C, D, E, F]$ denotes the Fourier transform of $[a, b, c, d, e, f]$ given in (16), respectively.

Finally, by solving the Fourier transform of (16) and substituting its closed form expressions into (17), we get (6).

This completes our Proof 1.

It is important to note that expression (6) for the Wigner time-frequency spectrum describes the temporal evolution of the phase-noise spectrum instantaneously. The power spectrum $S_{\Delta \varphi}(f)$ of the phase noise is obtained by integration of the resulted Wigner spectrum (6) within a temporal time "window" $T$, an operation which is "smoothing" short-term nonstationary instabilities in the phase variations:

$$
\begin{aligned}
S_{\Delta \varphi}(f) & =\lim _{T \longrightarrow \infty} \frac{1}{T} \int_{-T / 2}^{T / 2} \bar{W}_{\Delta \varphi}(t, f) d t \\
& =\left(2 \pi v_{0}\right)^{2}\left[\frac{\sigma_{1}^{2}}{4(\pi f)^{2}}+\frac{\sigma_{2}^{2}}{(2 \pi f)^{4}}\right] .
\end{aligned}
$$

A random process is said to be stationary (in a strictsense) if all its statistical properties are invariant under an arbitrary time shift, that is, the autocorrelation function depends only on the time difference. Therefore, by using the Wiener-Khintchine theorem, the resulted power spectrum $S(f)$ of a stationary process is dependent only on the frequency $f$ (without any temporal variations).

Please note that the integration of (6) in a finite observation time $T$ produced a frequency dependent power spectrum as expected in stochastic processes, which are wide-sense stationary.

Inspection of equation (18) reveals two different stationary noise processes;

The first term describes the white frequency noise which declines by $f^{-2}$ power law while the second term describes the random walk frequency noise which declines by $f^{-4}$ power law [35, 38].

An estimation of the nonstationary characteristics of (6) can be made by fitting the two noise sources expressed in (18) with the power law function $S_{\varphi}(f)=\sum b_{i} f^{i}$ for the phase noise spectra [38]. Please note that preforming the estimation gives $b_{-2}=2 \nu_{0} \sigma_{1}^{2}$ and $b_{-4}=\left(v_{0}^{2} \sigma_{2}^{2} / 2 \pi^{2}\right)$, which is exactly the results for $b_{-2}$ and $b_{-4}$ coefficients derived in [35].

\section{Numerical Simulations}

The Wigner-Ville distribution can be used to identify shortterm, nonstationary instabilities in the oscillator phase and follow its spectral evolution in time. Differing from the power spectral density, which is a result of a time assimilation, assuming a stationary behavior within the temporal integration window, the Wigner-Ville distribution is a fundamental time-dependent measure, which can trend phase variations in ultrahigh resolution, depending on the sampling rate only. We employ the Wigner-Ville spectrum to analyse the nonstationary phase noise of a high accuracy Rubidium atomic clocks. Two clock models are used in the experiments: AR13300 [43] and NAC1 [44]. These atomic clocks are designed to generate a harmonic signal at frequency of $v_{0}=10 \mathrm{MHz}$.

While the NAC1 Rubidium resonator is locked on local Temperature-Compensated Crystal Oscillator (TCXO) and the AR13300 Rubidium resonator is locked on a local OvenControlled Crystal Oscillator (OCXO), and thus expected to be more stable (characterized with a lower diffusion parameter $\sigma_{1}$ ). Table 1 summarizes the atomic clocks characteristics of both models.

The two noise types, white frequency noise and the random walk frequency noise, which are characterized by $\sigma_{1}$ and $\sigma_{2}$, respectively, are those commonly encountered in the measurement of oscillator phase noise. The manufacturer predefined the total power spectrum $S_{\varphi}(f)$ and then $\sigma_{1}$ and $\sigma_{2}$ magnitudes extracted [38].

In Figures 1 and 2, we present a three-dimensional plot of the calculated phase-noise Wigner spectrum of the $\mathrm{Ru}-$ bidium-based TCXO (model NAC1) and the Rubidiumbased OCXO (model AR13300) atomic oscillators, respectively. The temporal spectral variations of the OCXO based are revealed to be smaller than that of the NAC1 model. Integration of the Wigner spectrum in time, results in the power spectral density $S_{\Delta \varphi}(f)$, as shown in Figure 3. A comparison is made between the NAC1 and the AR13300. The last is shown to be more stable as expected.

\section{Summary and Conclusion}

In this letter, we presented an effective technique for phase noise characterization of oscillators in time-frequency domain. While the common measure of phase noise power spectrum fails to follow nonstationary processes, the Wigner-Ville distribution is shown to demonstrate highaccuracy analysis of short time phase instabilities and a comprehensive description of their evolution in high levels of temporal resolution. It is shown that the Wigner-Ville distribution can be utilized for a nonstationary phase noise characterization that can reveal time-varying spectrum, generally seen in the oscillators and clocks, during fast variating conditions, including vibrations, shocks, and 
TABLE 1: Summary table of the diffusion parameters vs. the oscillator type $[43,44]$ and its floor phase noise.

\begin{tabular}{lcccc}
\hline Oscillator & Model & $\sigma_{1}$ & $\sigma_{2}$ & Approximate phase noise in $10 \%$ frequency shift \\
\hline Rubidium-based OCXO & AR13300 & $10^{-4}$ & $10^{-6}$ & $-160 \mathrm{dBc} / \mathrm{Hz}$ \\
Rubidium-based TCXO & NAC1 & $2 \cdot 10^{-4}$ & $10^{-6}$ & $-150 \mathrm{dBc} / \mathrm{Hz}$ \\
\hline
\end{tabular}

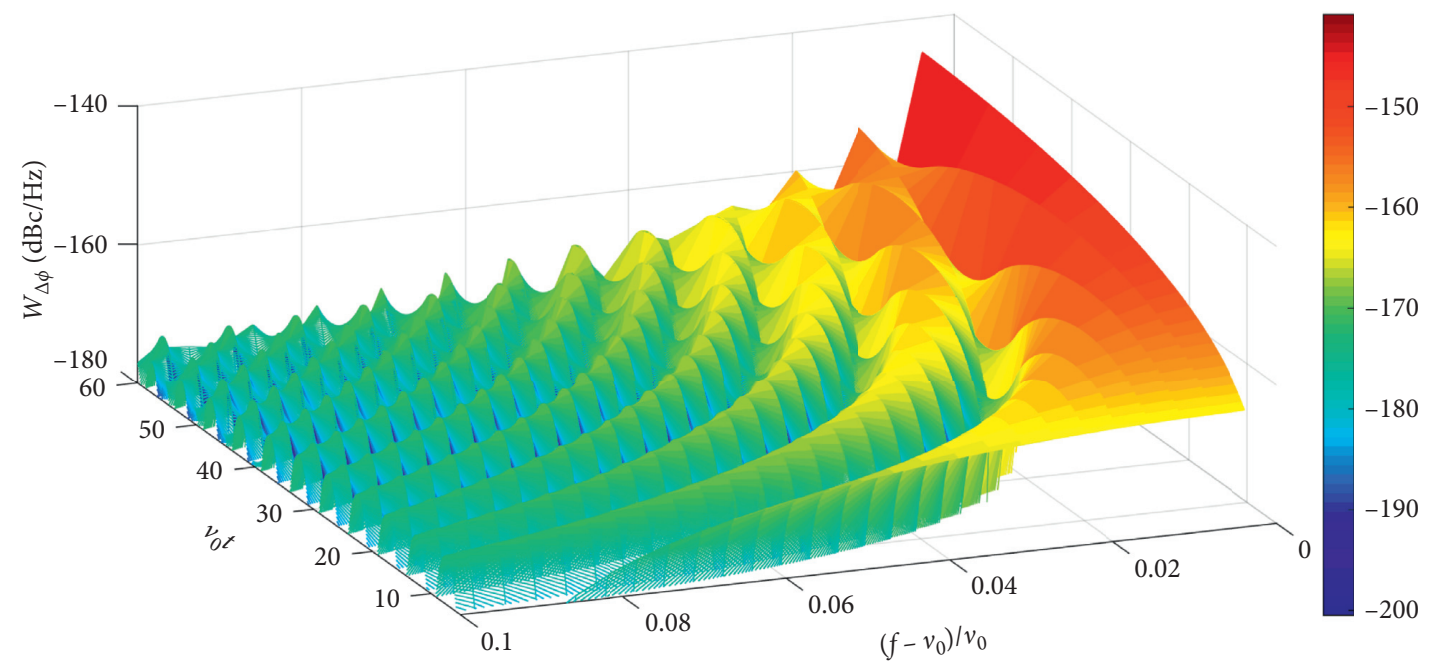

Figure 1: The analytic Wigner-Ville time-dependent spectrum $\bar{W}_{\Delta \varphi}(t, f)$ of NAC1 atomic oscillator.

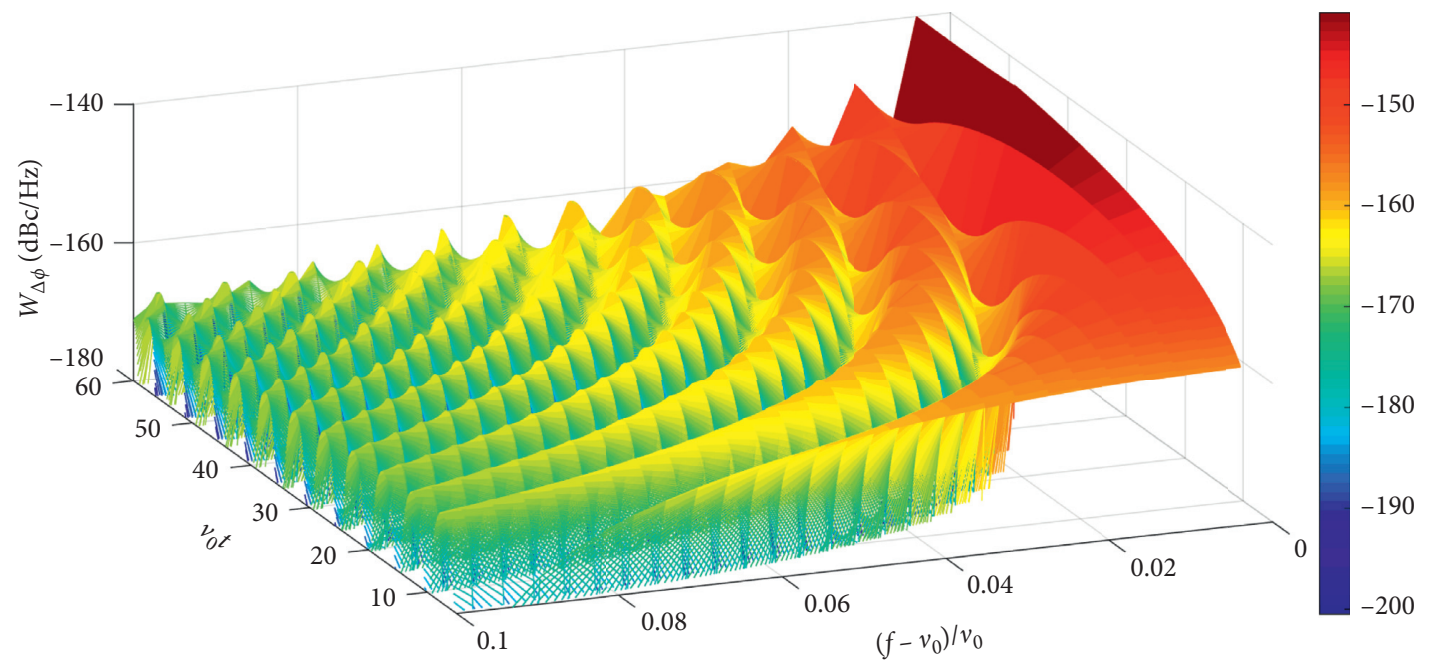

FIGURE 2: The analytic Wigner-Ville time-dependent spectrum $\bar{W}_{\Delta \varphi}(t, f)$ of AR13300 atomic oscillator.

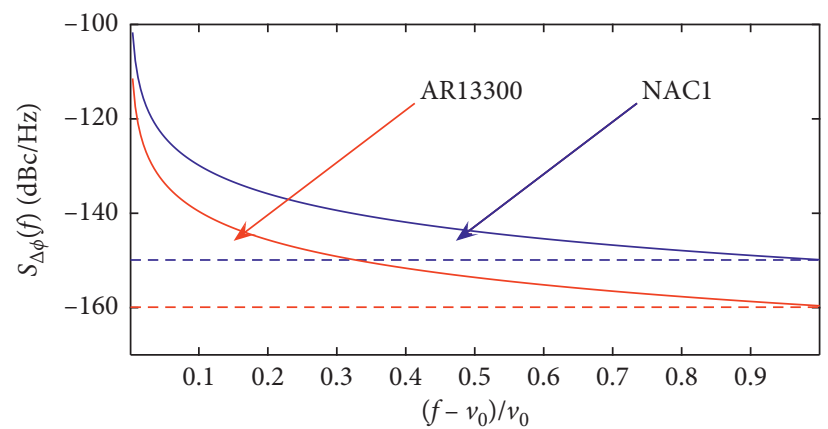

FIgURE 3: The power spectrum phase-noise $S_{\Delta \varphi}(f)$ of NAC1 and AR13300 obtained by integration of the resulted Wigner spectrum $\bar{W}_{\Delta \varphi}(t, f)$. 
temperature variations. We derived an analytic expression for the Wigner-Ville spectrum for nonstationary phase noise and demonstrate its application on atomic clocks. The technique is applicable for the characterization of different oscillators, frequency generators, and radiation sources including lasers and masers.

\section{Data Availability}

The data used to support the findings of this study are included within the article.

\section{Conflicts of Interest}

The authors declare that they have no conflicts of interest.

\section{References}

[1] T. Krawinkel, S. Schn, and A. Bauch, "Application of miniaturized atomic clocks in kinematic gnss single point positioning," in Proceedings of the 2014 European Frequency and Time Forum (EFTF), Neuchatel, Switzerland, June 2014.

[2] Y. Jin, B. Luo, X. Hu, and P. Zheng, "A Fast Positioning Method Based on High-Precision Clock for GNSS Receivers," in Proceedings of the 2015 IEEE International Conference on Information and Automation, Lijiang, China, August 2015.

[3] Internationational Standard, Timing and Synchronization for Time-Sensitive Applications in Bridged Local Area Networks, Internationational Standard, Geneva, Switzerland, 2011.

[4] R. Mikinis, D. Smirnov, E. Urba, and B. Dzindzelta, “Timing and Synchronization in Mobile Telecommunication Networks," 2011.

[5] L. Galleani and S. Member, "IEEE, and patrizia tavella, senior member, detection of atomic clock frequency jumps with the kalman filter," IEEE Transactions on Ultrasonics, Ferroelectrics, and Frequency Control, vol. 59, no. 3, 2012.

[6] L. Galleani and P. Tavella, "The characterization of clock behavior with the dynamic Allan variance," in Proceedings of the IEEE IFCS and European Frequency and Time Forum, Keystone, CL, USA, August 2003.

[7] I. Sesial, Galleani, and P. Tavella, "Application of the dynamic allan variance for the characterization of space clock behavior," IEEE Transactions on Aerospace and Electronic Systems, vol. 47, no. 2, 2011.

[8] J. Rutman and F. L. Walls, "Characterization of frequency stability in precision frequency sources," IEEE, vol. 79, no. 7, pp. 952-960, 1991.

[9] J. R. Vig and S. Eva, "IEEE standard definitions of physical quantities for fundamental frequency and time metrology random instabilities," IEEE, vol. 26, 1999.

[10] Z. H. Peng, Y.-X. Liu, J. T. Peltonen, T. Yamamoto, J. S. Tsai, and O. Astafiev, "Correlated emission lasing in harmonic oscillators coupled via a single three-level artificial atom," Physical Review Letters, vol. 115, 2015.

[11] S. Kim, Bo Zhang, Z. Wang et al., "Coherent polariton laser," Physical Review Letters, vol. 6, 2016.

[12] M.-G. Suh, Q.-F. Yang, and K. J. Vahala, "Phonon-limitedLinewidth of brillouin lasers at cryogenic temperatures," Physical Review Letters, vol. 119, 2017.

[13] N. Y. Voo, P. Horak, M. Ibsen, and W. H. Loh, “Anomalous linewidth behavior in short-cavity single-frequency fiber lasers," IEEE Photonics Technology Letters, vol. 17, no. 3, pp. 546-548, 2005.
[14] P. Gallion, H. Nakajima, G. Debarge, and C. Chabran, "Contribution of spontaneous emission to the linewidth of an injection-locked semiconductor laser," Electronics Letters, vol. 21, no. 14, pp. 626-628, 1985.

[15] M. P. van Exter, S. J. M. Kuppens, and J. P. Woerdman, "Excess phase noise in self-heterodyne detection" IEEE Journal of Quantum Electronics, vol. 28, no. 3, 1992.

[16] W. Yang, "Linewidth enhancement factor simplified," in Proceedings of the IEEE Southeast Conference 2010 (SoutheastCon), Concord, NC, USA, 2010.

[17] Y.-J. Cheng, P. L. Mussche, and A. E. Siegman, "Measurement of laser quantum frequency fluctuations using a PoundDrever stabilization system," IEEE Journal of Quantum Electronics, vol. 30, no. 6, pp. 1498-1504, 1994.

[18] L. Cohen, Time-Frequency Analysis, Prentice-Hall, Upper Saddle River, NJ, USA, 1994.

[19] E. Wigner, "On the quantum correction for thermodynamic equilibrium," Physical Review, vol. 40, no. 5, pp. 749-759, 1932.

[20] R. Torres and E. Torres, "Fractional fourier analysis of random signals and the notion of \$ \alpha-Stationarity of the wignerville distribution," IEEE Transactions on Signal Processing, vol. 61, no. 6, pp. 1555-1560, 2013.

[21] J. M. O' Toole, M. Mesbah, and B. Boashash, "Improved discrete definition of quadratic time-frequency distributions," IEEE Transaction Signal Process, vol. 58, no. 2, pp. 906-911, 2010.

[22] X. Lv, G. Bi, C. Wan, and M. Xing, "Lv's distribution: principle, implementation, properties, and performance," IEEE Transactions on Signal Processing, vol. 59, no. 8, pp. 3576-3591, 2011.

[23] P. Flandrin and P. Borgnat, "Time-frequency energy distributions meet compressed sensing," IEEE Transactions on Signal Processing, vol. 58, no. 6, pp. 2974-2982, 2010.

[24] T. O. Gulum, P. E. Pace, and R. Cristi, "Extraction of polyphase radar modulation parameters using a Wigner-Ville distribution-Radon transform" in Proceedings of the IEEE International Conference on Acoustics, Speech and Signal Processing, pp. 1505-1508, New York, NY, USA, 2008.

[25] Y. Wu, C. David, and A. Munson Jr., "Multistatic passive radar imaging using the smoothed pseudo wigner-ville distribution" in Proceedings of the International Conference on Image Processing, Thessaloniki, Greece, September 2001.

[26] S. Gabarda, G. Cristobal, J. Martnez-Alajarn, and R. RuizMerino, "Detection of anomalous events in biomedical signals by wigner analysis and instant-wise Rnyi entropy," in Proceedings of the 2006 14th European Signal Processing Conference, Florence, Italy, September 2006.

[27] F. Akdeniz, K. Kayikiolu and T. Kayikiolu, "Using wignerville distribution in ECG arrhythmia detection for telemedicine applications," in Proceedings of the 39th Telecommunications and Signal Processing (TSP) International Conference on IEEE, pp. 409-412, Vienna, Austria, June 2016.

[28] F. A. Kayikiolu and T. Kayikiolu, "Wigner-Ville Distribution Based ECG Arrhythmia Detection for Telemedicine Applications," in Proceedings of the 24th Signal Processing and Communication Application Conference (SIU), pp. 2045-2048, Zonguldak, Turkey, May 2016.

[29] S.-C. Pei and J.-J. Ding, "Fractional fourier transform, wigner distribution, and filter design for stationary and nonstationary random processes," IEEE Transactions on Signal Processing, vol. 58, no. 8, pp. 4079-4092, 2010.

[30] G. Boudreaux-Bartels and T. Parks, “Time-varying filtering and signal estimation using Wigner distribution synthesis 
techniques," IEEE Transactions on Acoustics, Speech, and Signal Processing, vol. 34, no. 3, pp. 442-451, 1986.

[31] L. Yang, L. Zhao, S. Zhou, and G. Bi, "Sparsity-driven SAR imaging for highly maneuvering ground target by the combination of time-frequency analysis and parametric bayesian learning," IEEE Journal of Selected Topics in Applied Earth Observations and Remote Sensing, vol. 10, no. 4, pp. 1443$1455,2017$.

[32] J. Alirezaie and J. Tavakkoli, "Shear wave elastography using wigner-ville distribution: a simulated multilayer media study," in Proceedings of the 2016 38th Annual International Conference of the IEEE Engineering in Medicine and Biology Society (EMBC), pp. 2873-2876, Orlando, FL, USA, 2016.

[33] D. Pang, P. Zhao, and B. Deng, "Cross-terms suppression in Wigner-Ville distribution based on image processing" in Proceedings of the 2010 IEEE International Conference on Information and Automation, pp. 2168-2171, Harbin, China, 2010.

[34] Keysight Technologies, "Analyzing frequency stability in the frequency and time domains," Keysight Technologies, Santa Rosa, CA, USA, 2014.

[35] C. Zucca and P. Tavella, "The clock model and its relationship with the Allan and related variances," IEEE Transactions on Ultrasonics, Ferroelectrics and Frequency Control, vol. 52, no. 2, pp. 289-296, 2005.

[36] C. Zucca, "A mathematical model for the atomic clock error in case of jumps," 2015.

[37] A. J. Van Dierendonck, J. B. McGraw, and R. Grover Brown, "Relationship between allan variances and kalman filter parameters," in Proceedings of the Sixteenth Annual Precise Time and Time Interval (PTTI) Applications and Planning Meeting, pp. 27-29, Greenbelt, MD, USA, 1984.

[38] E. Rubiola, Phase Noise and Frequency Stability in Oscillators, Cambridge University Press, Cambridge, UK, 2009.

[39] J. Ville, "Theorie et applications de la notion de signal analytique," Cables et Transmission, vol. 2, no. 1, pp. 61-74, 1948.

[40] W. Martin and P. Flandrin, "Wigner-Ville spectral analysis of nonstationary processes," IEEE Transactions on Acoustics, Speech, and Signal Processing, vol. 33, no. 6, pp. 1461-1470, 1985.

[41] F. C. Klebaner, Introduction to Stochastic Calculus with Applications, Imperial College Press, London, UK, 1998.

[42] E. Steven, Shreve, Stochastic Calculus for Finance II: Continuous-Time Models, Springer, Berlin, Germany, 2004.

[43] http://www.accubeat.com/product-item/ar133a/.

[44] http://www.accubeat.com/product-item/nano-atomic-clocknac1/. 